\title{
Application of Blended Learning on the Antenatal Education Center Course: A Review of Flipped Classroom with Team-based Learning
}

\section{Yingying Lin}

Affiliated Hospital of Fujian Medical University

\section{Xiaoyan Xiu}

Affiliated Hospital of Fujian Medical University

Juan Lin

Affiliated Hospital of Fujian Medical University

Zhiwei Chen

Affiliated Hospital of Fujian Medical University

Cui Xian Zheng

Affiliated Hospital of Fujian Medical University

Xuehong Pan

Affiliated Hospital of Fujian Medical University

Lihua Lin

Affiliated Hospital of Fujian Medical University

Jianying Yan ( $\nabla$ yanjy2019@fjmu.edu.cn )

Affiliated Hospital of Fujian Medical University

\section{Research Article}

Keywords: Health education, Flipped classroom, Team-based learning, Primipara, Pregnancy outcomes

Posted Date: June 25th, 2021

DOI: https://doi.org/10.21203/rs.3.rs-640411/v1

License: (c) (1) This work is licensed under a Creative Commons Attribution 4.0 International License. Read Full License 
1 Application of Blended Learning on the Antenatal Education Center Course: a

2 review of Flipped Classroom with Team-based Learning

3

4 Yingying Lin ${ }^{1 \#}$, Xiaoyan Xiu ${ }^{2 \#}$, Juan $\operatorname{Lin}^{3}$, Zhiwei Chen ${ }^{3}$, Cui Xian Zheng ${ }^{3}$, Xuehong $5 \quad \operatorname{Pan}^{2}$, Lihua Lin ${ }^{1}$, Jianying Yan $^{3 *}$

$6 \quad{ }^{1}$ Department of Healthcare, Fujian Provincial Maternity and Child Health, affiliated 7 hospital of Fujian Medical University, Fuzhou, P. R. of China;

$8{ }^{2}$ Department of Health Education, Fujian Provincial Maternity and Child Health, 9 affiliated hospital of Fujian Medical University, Fuzhou, P. R. of China;

$10{ }^{3}$ Department of Obstetrics, Fujian Provincial Maternity and Child Health, affiliated 11 hospital of Fujian Medical University, Fuzhou, P. R. of China;

"These authors have contributed equally to this work.

* Corresponding Author:

Jianying Yan, M.D, Professor, Fujian Provincial Maternity and Child Health, Affiliated Hospital of Fujian Medical University, 18 Daoshan Road, Fuzhou 350001, Fujian, P.R. China

Email: yanjy2019@fjmu.edu.cn

Tel: 0086-13705025141 


\begin{abstract}
Background: The goal is to evaluate the effects of a flipped class strategy on knowledge, self-directed learning ability, learning satisfaction and pregnancy outcomes in primiparas undergoing antenatal education.
\end{abstract}

Methods: A convenience sampling method was adopted. A total of 621 primiparas who were diagnosed with early pregnancy in a first-class hospital in southeast China and received continuous prenatal health education from May to July 2020 were selected as the research subjects. The primiparas were divided into two groups in the antenatal education centre. One was the experimental group, who used the prenatal health education model based on blended learning (a flipped classroom with team-based learning); the other was the control group, who used the traditional mode of prenatal health education. The two groups were compared on the following outcomes: knowledge, self-directed learning ability, learning satisfaction and pregnancy outcomes.

Results: The blended learning approach enhanced the primiparas' knowledge, self-directed learning ability, and learning satisfaction, effectively controlled the gestational weight gain (GWG), alleviated the degree of anxiety and depression in the pregnant women during pregnancy, improved the self-efficacy and the natural delivery rate of the primipara, shortened the delivery process and reduced the risk of gestational diabetes mellitus (GDM), all $P<0.05$.

Conclusions: Blended learning may be an effective strategy because of its validity and practicality in antenatal education.

Keywords: Health education, Flipped classroom, Team-based learning, Primipara, Pregnancy outcomes

\title{
Background
}

Maternal health education in China is based on antenatal education centres supported by health care institutions at all levels. The "Regulations for Pregnancy and Childbirth Health Care" issued by the former Ministry of Health of the People's Republic of China clearly stipulates that "Maternal and child health institutions at all levels are responsible for guiding and carrying out pregnancy and childbirth health education in their respective jurisdictions, formulating a health education plan and developing suitable health education materials" ${ }^{[1]}$. As an important component of perinatal health care work, antenatal education centres play a vital role in publicizing and promoting perinatal health care knowledge.

However, with the advent of social media, the new generation of prospective parents are seeking increasingly different kinds of information about pregnancy on the Internet during early gestation. Studies have shown that the Internet is widely used not only as a source of information but also for pregnancy-related social networking and support and that it has a significant impact on the prenatal and postpartum decision-making process ${ }^{[2]}$. Pregnant women are more actively participating in their entire pregnancy. Therefore, the traditional lecture (TL) model obviously can no longer meet the needs of contemporary pregnant women.

More interactive prenatal education models are being developed and utilized, for example, the application of learner-centred education and active participation of 
learners in the classroom ${ }^{[3,4]}$. The American Board of Obstetrics and Gynaecology advocates the group prenatal care (GPC) model ${ }^{[5]}$. Based on this model, several pregnant women with similar gestational weeks form a team and acquire antenatal care together and regularly organize activities for discussion and the publicizing of knowledge. On the team, pregnant women can grow up to each other and enhances the quality of prenatal care ${ }^{[6]}$. Such educational backgrounds have stimulated the development of various teaching patterns and learning strategies, such as the flipped classroom (FC) method and team-based learning (TBL).

The FC method covers pre-, in-, and post-class learning methods. First, the instructor provides various learning materials through an electronic network. Learners acquire foundational knowledge by means of various digital media, such as their cell phones or laptops, at any time and in any location. Second, knowledge is transferred from the instructor to the learners through this media. Third, learners apply this knowledge in the classroom ${ }^{[7,8]}$. The FC may encourage learners to think independently and imaginatively, but it appears that learners perform only limited pre-learning before class $^{[9]}$. TBL is a teaching strategy that involves the instructional use of small teams and transforms such teams into active learning teams by specific procedures ${ }^{[10]}$. It can not only improve learners' collaboration ability, disciplinary knowledge and application ability ${ }^{[11]}$ but also has the potential to increase learners' satisfaction and achievement $^{[12]}$. Therefore, a blended learning model (i.e., a combination of FC and TBL) may promote learners' active learning. It could enhance learners' learning achievements by enabling them to learn individually and iteratively, facilitating the sharing of learning materials in groups, and helping them acquire high knowledge scores $^{[13,14]}$. Rather than completing tasks outside the classroom, learners apply TBL inside the FC. This increases the opportunity for active instructor-learner and learner-learner interactions, thereby improving learners' learning ability ${ }^{[15,16]}$. Several pedagogical theories, such as Piaget's active learning theory, have proven that a learner's interest in collaborative interaction for TBL motivates self-directed learning in the $\mathrm{FC}^{[8]}$. Active participation in the learning process also helps improve learners' learning satisfaction ${ }^{[17]}$.

At present, studies have just started to investigate the effect of the blended learning approach in antenatal education courses. The objection of this study is to fill this void in the field and evaluate the effect of the blended learning model in an antenatal education course in regard to learning outcomes and to the maternal and neonatal outcomes of primiparas undergoing antenatal education.

\section{Methods}

\section{Design and participants}

In this study, 621 pregnant women receiving antenatal education in the hospital from May to July 2020 were selected as the research subjects. To control any bias, this study stipulated that the primiparas must have no experience in TBL and FC approaches in their first trimester (11 weeks gestation). They were willing to participate in this study, able to attend regular antenatal examinations, planned to deliver in the target hospital, signed the informed consent form and had a smartphone. Exclusion criteria were refusal to participate in the study, language barriers, history of 
major disease or mental illness, employment as medical staff, high-risk pregnancy factors, or a psychological assessment of severe anxiety or depression. We assessed the respondents' psychiatric symptoms using the Self-Rating Anxiety Scale (SAS) and Self-Rating Depression Scale (SDS). At the end of the study, 11 patients in the control group were lost to follow-up, for a loss rate of $3.54 \%$. The data showed that 5 were withdrawals, 3 were miscarriages and 2 prematurely delivered. In the experimental group, 10 patients were lost to follow-up, with a loss rate of $3.33 \%$. The reasons for the loss to follow-up were 6 cases of withdrawal, 2 cases of miscarriage and 2 cases of premature delivery. Ultimately, 300 patients in each group completed this study, and the data were complete and effective.

\section{Instruments}

The general information questionnaire was designed by the researchers for the study and included the age, height, weight, educational background, and per capita monthly income of each family.

To test the knowledge of participants, we developed a 35-item multiple-choice questionnaire addressing nutrition during pregnancy, body quality control, exercise during pregnancy, delivery mode, breastfeeding, neonatal health care, infant health care and other related information. The questionnaire's total scores ranged from 0 to 35. The Cronbach's $\alpha$ value of the scale was 0.90 .

The self-directed learning ability scale developed by the Teaching and Learning Center of Fujian Medical University was used, which comprises 28 items to be answered on a five-point Likert scale (ranging from $1=$ strongly disagree to $5=$ strongly agree). The average value was calculated, and a higher score means better self-directed learning ability. The Cronbach's $\alpha$ value of the scale was 0.96 .

A standardized scale of the hospital's Teaching and Learning Center was used to measure learning satisfaction. Furthermore, the scale was regularly reviewed by a special academic advisory committee of the hospital. This scale comprises 13 items to be answered on a 4-point Likert scale (ranging from $1=$ strongly disagree to $4=$ strongly agree). The average value was calculated, and the higher the values were, the better the learning satisfaction. In this study, the Cronbach's $\alpha$ value of the scale was 0.93 .

Childbirth self-efficacy was measured using the short form of the Chinese Childbirth Self-Efficacy Inventory (CBSEI-C32), which was translated and developed by Wan-Yim IP in 2008. The scale has 32 items, including two subscales of outcome expectation (OE-16) and self-efficacy expectation (EE-16), each with 16 items, and uses a Likert scale of 10 , where 1 is not helpful/not sure at all and 10 is very helpful/very sure. This scale has good reliability and validity, with a Cronbach's $\alpha$ coefficient of $0.96^{[18]}$.

The self-efficacy of breastfeeding was measured using the Breastfeeding Self-efficacy Scale (BSES), which was introduced into China in 2002 by Xiaona Dai ${ }^{[19]}$ and forms two dimensions (skill dimension and inner activity dimension), for a total of 30 items. A Likert scale of 5 was adopted, with the numbers 1-5 representing "not confident at all" to "always confident", and the total score was 30-150. The higher the score was, the better the self-efficacy of breastfeeding. Overall, the scale has good structural 
validity, with a Cronbach's $\alpha$ coefficient of 0.93 .

The SAS developed by Professor Zung in 1965 was adopted for anxiety status. The scale contains 20 items, and the appropriate option is selected to fill in according to the actual feeling of the respondents during the recent week. It adopts a Likert scale of 4: there are 15 SAS sentences with negative words, scoring in order from 1 to 4 , the remaining five positive word statements are reverse scored from 4 to 1 . The total rough score was calculated according to the options and then converted to a standard score according to the standard score conversion table. Anxiety status was determined by a SAS standard score above 50, which indicates anxiety ${ }^{[20]}$.

The SDS developed by Professor Zung in 1971 was adopted for depression status. The scale contains 20 items. The actual feelings of the respondents were used to evaluate their depression status in the last week. A Likert scale of 4 was used to calculate the total rough score according to the options, which was then converted to a standard score for evaluation. Depression status was determined by an SDS standard score greater than 53, which indicates depression ${ }^{[21]}$.

\section{Data collection and procedures}

This study was approved by the Ethics Committee of Fujian Maternity and Children's Health Hospital, Affiliated Hospital of Fujian Medical University. All individuals participating in this study provided written informed consent. According to the protocol approved by the Research Ethics Committee, participation (or lack of participation) would not influence their clinical care. The research team earnestly protected the rights and interests of the research subjects, such as privacy, respect and knowledge, strictly kept all data confidential, and carried out anonymous numbering for the included samples. The researchers used neutral language and a non-judgmental attitude throughout the study.

\section{Blended learning design}

Figure 1 depicts the process of FC combined with TBL education reform. The process included reviewing previous research and on the blended learning design, the application of blended learning and effect evaluation. The teaching process was divided into three stages: pre-class preparation, in-class learning and post-class reflection. The information was delivered once every two weeks in a one-hour class for a total of 12 classes.

One week before class, the instructor provided the learners with the relevant learning materials using the WeChat platform. The learners' WeChat group was set up by dividing the learners into 10 groups and selecting a leader for each group. Learners in small groups previewed the learning materials before class and conducted independent learning to familiarize themselves with the relevant teachings and consider the key points and difficulties of the lesson. Before class, the instructor organized a discussion within the group to exchange knowledge or ask questions to improve the quality of the preview. After the discussion, the group leader summarized the questions and key difficult points in the class. During the class stage, the instructor first evaluated the preview effect, then gave a 20-minute lecture, and finally played a demonstration video. Taking groups as units, learners carried out the practical operation of exercises under the guidance of group instructors. Two learners from 
each group were randomly selected to demonstrate the operation and one of them gave a presentation. The other learners observed and commented, and finally, the instructor summarized the key points and difficulties of the lesson. During the after-class reflection stage, the learners took test questions, and according to the test results and their performance in class, the instructor formulated personalized learning modes and team activity suggestions. The instructor adjusted and reflected upon the teaching content, improved the teaching method, optimized the teaching design, enhanced the teaching effect and enhanced the interest of the learners.

\section{Traditional lecture}

The control group adopted traditional prenatal health education, which comprises fixed courses compiled according to knowledge of prenatal health education and organized according to the first trimesters (T1), second trimesters (T2) and third trimesters (T3). It is run in an antenatal education centre using a lecture based approach to prenatal health education. The information was again delivered once every two weeks in a one-hour class for a total of 12 classes.

Before starting the education intervention, participants in both groups completed questionnaires assessing their antenatal education-related knowledge, self-directed learning abilities, and learning satisfaction regarding the course that they had previously attended at 11 weeks of pregnancy in the antenatal education centre. Participants in both groups completed the same questionnaires during pregnancy.

\section{Statistical analysis}

SPSS 25.0 statistical software was used for data analysis, and $P<0.05$ was considered statistically significant. The measurement data are described by the mean $\pm \mathrm{SD}$, and the counting data are expressed by the frequency and composition ratio. A chi-square test and independent sample $t$ test were used for comparisons between the two groups. The differences between the two groups in self-directed learning ability, knowledge, learning satisfaction, gestational weight gain (GWG), SAS score and SDS score in accordance with the intervention were analysed by one-way repeated measure ANOVA.

\section{Results}

\section{General characteristics of participants between the two groups}

A total of 600 primiparas were included. Table 1 shows the general characteristics. The average ages of the experimental group and control group were 28.83 and 28.66, respectively. There was no difference in age, weight, height, educational background or family per capita monthly income between the two groups $(P>0.05)$. They are comparable.

\section{Differences in learning outcomes and psychological tests between the two groups}

There was no difference in statistics between the two groups for psychological tests or the T1 learning outcome variables. The experimental group significantly improved all learning outcome scores and saw an alleviated degree of anxiety and depression in patients. By repeated ANOVA, the self-directed learning ability score showed statistically significant differences in the interactions between the groups $(\mathrm{F}=552.54$, $P<0.001)$, between the gestational week groups $(\mathrm{F}=6416.57, P<0.001)$, and between the groups and the gestational week groups $(\mathrm{F}=769.17, P<0.001)$. The knowledge 
score showed statistically significant differences in the interactions between the groups $(\mathrm{F}=122.96, P<0.001)$, between the gestational week groups $(\mathrm{F}=1873.40$, $P<0.001)$, and between the groups and the gestational week groups $(\mathrm{F}=275.71$, $P<0.001)$. The learning satisfaction score showed statistically significant differences in the interactions between the groups $(\mathrm{F}=24.55, P<0.001)$, between the gestational week groups $(\mathrm{F}=864.30, P<0.001)$, and between the groups and the gestational week groups $(\mathrm{F}=206.66, P<0.001)$. The SAS score showed statistically significant differences in the interactions between the groups ( $\mathrm{F}=233.42, P<0.001)$, between the gestational week groups $(\mathrm{F}=2281.41, P<0.001)$, and between the groups and the gestational week groups $(\mathrm{F}=155.79, P<0.001)$. The SDS score showed statistically significant differences in the interactions between the groups $(\mathrm{F}=26.32, P<0.001)$, between the gestational week groups $(\mathrm{F}=100.25, P<0.001)$, and between the groups and the gestational week groups $(\mathrm{F}=23.90, P<0.001)$ (Table 2).

\section{Differences in GWG and childbirth between the two groups}

GWG, delivery mode and newborn birth weight were significantly different between the two groups. The proportions of underweight gain during pregnancy and overweight gain during pregnancy in the experimental group were $18.00 \%$ and $22.00 \%$, respectively, which were lower than the $24.00 \%$ and $30.67 \%$ in the control group $\left(\chi^{2}=12.98, P=0.002\right)$. Using repeated ANOVA, the interaction effect of GWG in both groups was not statistically significant $(P>0.05)$. GWGs are different during different periods. Additionally, the GWG between the two groups was different, and the differences were statistically significant $\left(\mathrm{F}_{\text {time }}=3026.145, \quad P_{\text {time }}<0.001\right.$; $\mathrm{F}_{\text {groups }}=9.463, P_{\text {groups }}=0.002$ ). A multivariate ANOVA was used, and the results showed that there was no significant difference in the T1 of GWG in either group $(\mathrm{P}>0.05)$, but the T2, T3 and total GWG in the study group were all lower than those of the control group (F values were 4.43, 14.08 and 9.46, $\mathrm{P}$ values were all $<0.05$ ). Compared to the control group, the incidence of natural childbirth increased, while the incidence of caesarean section decreased. There were obvious differences in both groups $\left(\chi^{2}=6.51, P=0.039\right)$. The incidence of low birth weight and macrosomia in the experimental group was $3.30 \%$ and $1.70 \%$, respectively, which were significantly lower than the $6.70 \%$ and $4.70 \%$ in the control group $\left(\chi^{2}=8.25, P<0.05\right)$. However, the gestational age was not significantly different between the two groups $(P>0.05)$ (Tables 3-4).

\section{Differences in delivery time in both groups}

The delivery times of the first, second, and the total stage of labour in the experimental group were significantly less than those of the control group ( $P$ values were all $<0.001)$, but there was no significant difference in the delivery time of the third stage of labour between the two groups $(P>0.05)$ (Table 5).

\section{Differences in the self-efficacy of both groups}

The test scores for the CSE and BSE of primiparas in the experimental group were 269.66 and 136.17, respectively, which were higher than the 203.10 and 102.03 in the control group, and the difference were statistically significant $\left(t_{C S E}=80.88, P<0.001\right.$; $t_{B S E}=70.62, P<0.001$ ) (Table 6).

\section{Differences in complications during childbirth between the two groups}


The rate of GDM was $16.3 \%$ in the study group, which was significantly lower than the control group's $23.0 \%$, and there was a significant difference $\left(\chi^{2}=4.220, P=0.040\right)$. Other factors, such as PH, HDCP and intrauterine distress, were not significantly different between the groups $(P>0.05)$ (Table 7).

\section{Discussion}

\section{The application of a blended learning model in antenatal education}

With the decline in traditional methods of information sharing, structured antenatal classes have been developed worldwide, driven by the expectations of those who are preparing for childbirth and parenthood ${ }^{[22,23]}$. The dissemination of antenatal information is constantly changing to meet the needs and expectations of parents. Antenatal education should be an educational intervention but not an obstetric intervention. Blended learning is consistent with the core idea of constructivist theory. Namely, knowledge is not taught by teachers but acquired by learners through meaning construction with the help of teachers, learning partners and necessary learning materials in the learning process under certain social and cultural conditions. Our study suggests that blended learning can enhance outcomes in antenatal education and improve autonomous learning ability, knowledge level, learning satisfaction and even the conditions of the mother and the baby. There are limited studies on the use of a blended learning strategy in antenatal education centres. This article aims to explore the application of a blended learning model in antenatal health education.

2. The influence of antenatal education for primiparas based on flipped classrooms on learning outcomes

The results of Table 2 show that compared with traditional antenatal education, blended learning can significantly improve the actual effect of antenatal education for primiparas, including improving their knowledge level, learning satisfaction and self-learning ability.

First, the knowledge score of the experimental group was significantly higher than that of the control group, indicating that the flipped classroom has advantages over the traditional classroom in deepening the primiparas' comprehensive understanding and analysis of knowledge. As traditional antenatal education is teacher-centred, primiparas are more passive in learning knowledge, ignoring the process of active exploration and constructive learning. However, the flipped classroom treats primiparas as the centre, and its unique three-process model is more helpful for expanding primiparas' knowledge of pregnancy than the TL model. Primiparas learn and ask questions according to their own situation before class, fully communicate with teachers to solve difficult problems in class, and apply their knowledge and skills to try to solve practical problems after class, thus further strengthening their understanding and application of pregnancy knowledge.

Second, compared with traditional classrooms, flipped classrooms can significantly improve pregnant women's satisfaction. Learner-centred discussions and learner facilitating behaviours expressed through learning activities help participants feel valued in a learning environment ${ }^{[24,25]}$. Interactive discussion is carried out in a flipped classroom. On the one hand, the instructors sort out the knowledge and show their thought process by answering questions in a guiding manner; on the other hand, 
the primiparas actively participate and think throughout the process. Thus, primiparas have a better learning experience and a stronger interest in learning. Moreover, flipped classrooms can meet the learning needs of pregnant women in a timely manner. Primiparas receive customized learning materials through WeChat Official Accounts. If they encounter problems in learning, they can communicate with the research team through a mobile network immediately. Last, but not least, team education makes enjoyable learning possible. Through group interactive learning, primiparas gain in-depth knowledge and experience exchanges. The members of the group have similar experiences and can encourage each other and talk to each other when they encounter difficulties and puzzles in life. Petersson et al. found that participants had a positive understanding of knowledge exchange and experience sharing within a group ${ }^{[26]}$. Another study shows that women think informal support from their peers and professional support are important ${ }^{[27]}$. Peer support is considered to be an important and enjoyable component of antenatal education. Because of this, the flipped teaching mode obtains higher satisfaction.

Third, although the cultivation of autonomous learning ability is a complex systems engineering problem, flipped classrooms truly have advantages in cultivating primiparas' self-learning ability. The self-learning ability of primiparas in the flipped classroom group was significantly higher than that in the control group. In the blended learning model, learners are not limited by time or space before class, easily obtain various learning materials related to topics through smart devices, and realize self-learning. In the process of developing planning, learning and consulting materials, primiparas improved their self-management and self-control ability, and their self-learning awareness and thinking ability were improved.

\section{Antenatal education for primiparas based on flipped classrooms can shorten the delivery process and improve the natural childbirth rate and CSE}

The results of Table 3 show that the natural delivery rate of the flipped teaching group was higher than that of the control group, while the caesarean section rate was lower than that of the control group. This finding is consistent with the relevant research results $^{[28]}$. Table 6 shows that the delivery self-efficacy score of the experimental group was higher than that of the control group, which shows that antenatal education based on flipped classrooms can effectively improve the natural childbirth rate and childbirth self-efficacy of primiparas. CSE plays an important role in the success of natural childbirth, as it strengthens a woman's confidence in her ability to cope with childbirth. Higher levels of CSE are associated with lower levels of anxiety, pain, and obstetric intervention. Through innovative teaching methods, flipped classrooms strengthen primiparas' absorption and mastery of delivery knowledge and skills so that they can better understand the benefits of natural childbirth and the indications and sequelae of caesarean section. Some studies have proven that antenatal education in small groups may provide an environment that allows women to hear the experiences of other pregnant women and be encouraged by other participants and the group facilitator ${ }^{[27]}$. Through such methods, antenatal education in a small team may increase women's trust in their ability to cope with early childbirth, thus reducing the possibility of early admission and the experience of anxiety and childbirth pain 
[29,30,31]. As shown in Table 2, the flipped classroom can effectively improve the psychological evaluation results of primiparas, and thus it can improve the CES and natural childbirth rate of primiparas.

The results in Table 5 show that the times for the first, second and total stages of labour in the experimental group were lower than those in the control group. This shows that antenatal education based on flipped classrooms can shorten the labour process of primiparas. The flipped classroom model presents important free position, labour pain reduction methods, delivery auxiliary facilities and free position in the form of courseware and video, which can help primiparas master delivery skills effectively and eliminate stress. At the same time, the guidance of midwives is helpful for enhancing the self-confidence of primiparas and ensuring a smooth delivery process, thus shortening the time for the first stage, the second stage and the total course of labour .

\section{The influence of antenatal education of primiparas based on flipped classrooms on BSE}

BSE is a mother's expectation of her ability to breastfeed, and thus it is the determinant of her breastfeeding behaviour. Antenatal education for primiparas was based on the flipped classroom concept, and it sets a breastfeeding task list and regularly publicizes breastfeeding teaching resources. Primiparas can use available chunks of time to obtain breastfeeding knowledge, which is conducive to strengthening the internalization of breastfeeding knowledge. The TBL provides knowledge, emotion and skill support, such that primiparas shift from the passive acceptance of antenatal education knowledge to active participation in obtaining such knowledge. This is helpful in mastering the knowledge of breastfeeding and effectively improving the BSE of primiparas.

\section{The influence of antenatal education for primiparas based on flipped classrooms on weight gain during pregnancy, birth weight of newborns and GDM}

Women have an increased demand for nutrition and calories during pregnancy, and the balance of nutrition at all stages of pregnancy is very important for the health of primiparas. The results of Table 3 and Table 4 show that the GWG in the experimental group was lower than that in the control group regardless of the T2, T3 or pregnancy period. With reference to the guidelines for GWG revised by the Institute of Medicine (IOM) ${ }^{[32]}$, the proportions of overweight and underweight women in the experimental group were lower than those in the control group. The incidence of normal birth weight in the experimental group was higher than that in the control group, while the incidence of macrosomia and low birth weight was significantly lower than that in the control group. This shows that antenatal education for primiparas based on flipped classrooms can effectively control GWG during pregnancy, increase the birth rate of normal-weight newborns, and reduce the occurrence of huge babies.

This study uses a blended learning model to regularly publicize teaching resources to primiparas and inform them of the key points of body management at various stages of pregnancy, which can improve their cognition of GWG. A number of studies have pointed out that nutrition and health education for pregnant women can improve their 
nutritional awareness and change their unhealthy nutritional behaviours ${ }^{[33]}$. Nutritional intake and physical activity during pregnancy are the main factors affecting the occurrence of macrosomia. The blended learning model lists tasks related to individualized pregnancy nutrition, pregnancy activity and BMI management; it also offers individualized guidance to help primiparas establish a reasonable intake of energy and appropriate aerobic activities for primiparas during pregnancy to maintain their weight in a reasonable range. At the same time, blended learning strengthens the interaction between the primipara and the instructor and between the primiparas themselves, allowing individualized solutions to the problems of the primipara regarding body management and pregnancy activities that are timely, pertinent and scientific. In addition, primiparas who receive the blended learning model may pay more attention to the close relationship between the GWG and the weight of newborns. Therefore, this model can effectively control the GWG of primipara and reduce the occurrence of macrosomia.

The results in Table 7 show that blended learning can effectively reduce the incidence of GDM compared with traditional antenatal education. Nutritional imbalance at various stages of pregnancy may significantly increase the risk of pregnancy complications such as anaemia, hypertension syndrome, and GDM. A number of studies have found that GWG is closely related to $\mathrm{GDM}^{[34]}$. Excessive GWG will increase the mother's metabolic burden and lead to glucose and lipid metabolism disorders. The blended learning model actively guides pregnant women with reasonable nutrition, scientifically adjusts their diet and exercise, strengthens communication, solves nutritional problems in a timely manner, maintains blood sugar stability, and reasonably controls pregnant women's weight and GWG within an appropriate range, thereby reducing the risk of gestational diabetes.

\section{Limitations}

This study has some limitations. First, the study is limited to one region, with a small sample size and a lack of representativeness. Second, the current research only focuses on primiparas, but in reality, the proportion of multiparas can be as high as 44.4\%. Third, the current research period was less than one year, which limits understanding of long-term effects and other objective indicators, such as exclusive breastfeeding rate, child rearing and long-term health of mother and child.

At present, medical and health care institutions are relatively short of human resources, have a heavy workload, and devote less energy to health education. The smooth development of health education and health promotion is a national project that still needs support from the government and other relevant departments. The research area and sample size should be expanded in future research.

\section{Conclusions}

In short, the blended FC with TBL learning model enhanced primiparas' knowledge, self-directed learning ability, learning satisfaction, and effective GWG control and reduced depression in pregnant women during pregnancy, which improved the self-efficacy and natural delivery rate of the primipara, shortened the delivery process, and reduced the risk of GDM. In addition, this educational strategy was found to be effective both outside and inside the classroom in obtaining positive learning 
outcomes. The blended learning model presented in this research is remarkably effective and worth promoting.

\section{Abbreviations}

FC: Flipped classroom; TBL: Team-based learning; TL: Traditional lecture; SAS :Self-Rating Anxiety Scale; SDS: Self-Rating Depression Scale; CBSEI-C32: The short form of the Chinese Childbirth Self-Efficacy Inventory; BSES: Breastfeeding self-efficacy Scale; CSE: Childbirth self-efficacy; BSE: Breastfeeding self-efficacy; GWG: gestational weight gain; T1: First trimester; T2: Second trimester; T3: Third trimester; HDCP: hypertensive disorder complicating pregnancy; PH: Postpartum hemorrhage; GDM: gestational diabetes mellitus

\section{Acknowledgements}

We would like to thank all the women who kindly agreed to participate in this study. We are also grateful to all the staff at the hospitals and health facilities of the Fujian Maternity and Child Health Hospital and their support with this research.

\section{Authors' contributions}

YL, XX and JY was involved in the design of the study. JuanL, ZC, CZ, XP and LL were involved in data collection and analyzed the data. Both YL and XX were involved in drafting the manuscript, JY edit the manuscript. All authors have read and approved the final manuscript.

\section{Funding}

The study was supported by the National Key R\&D Program of China ( No.2016YFC1000102).

\section{Availability of data and materials}

Not applicable.

\section{Ethics approval and consent to participate}

This study was approved by the Ethics Committee of Fujian Provincial Maternity and Children Hospital ( FMCH2017-030) and complied with the Declaration of Helsinki. All individuals participating in this study provided written informed consent.

\section{Consent for publication}

Not applicable.

\section{Competing interests}

The authors declare that they have no competing interests.

\section{References}

1. Department of Maternal and Child Health and Community Health, Ministry of Health, PRC. "Regulations for Pregnancy and Childbirth Health Care" ([2011] No. 56) http://www.nhc.gov.cn/zwgkzt/wsbysj/201107/52320.shtml.

2. Moretti FA, Oliveira VE and Silva EM. 'Access to health information on the Internet: a public health issue?' Revista Assoc Med Brasileira, 58(6): 650-658.

3. O'Flaherty J, Phillips C. The use of flipped classrooms in higher education: a scoping review[J].Internet High Educ, 2015,25:85-95.

4. Mehta NB, Hull AL, Young JB, Stoller JK. Just imagine: new paradigms for medical education[J]. Acad Med, 2013,88(10):1418-23. 
5. ACOG committee opinion No. 731: group prenatal care[J].Obstet Gynecol, 2018, 131(3): e104-e108.

6. De Cesare JZ, Jackson JR. Centering pregnancy: practical tips for your practice[J]. Arch Gynecol Obstet, 2015,291(3): 499-507.

7. Bergmann J, Sams A. Flipped learning: Gateway to student engagement. 1st ed. Washington: International Society for Technology in Education, 2014.

8. Erbil DG. A review of flipped classroom and cooperative learning method within the context of Vygotsky theory[J].Front Psychol, 2020,11:1157.

9. Mortensen CJ, Nicholson AM. The flipped classroom stimulates greater learning and is a modern 21 st century approach to teaching today's undergraduates[J]. J Anim Sci, 2015,93(7):3722-31.

10. James S, Cogan P, McCollum M. Team-based learning for immunology courses in allied health programs[J].Front Immunol, 2019,10:2477.

11. Hamada S, Haruta J, Maeno T, Maeno T, Suzuki H, Takayashiki A, et al. Effectiveness of an interprofessional education program using team-based learning for medical students: a randomized controlled trial[J].J Gen Fam Med, 2019,21(1):2-9.

12. Dearnley C, Rhodes C, Roberts P, Williams P, Prenton S. Team based learning in nursing and midwifery higher education: a systematic review of the evidence for change[J].Nurse Educ Today, 2018,60:75-83.

13. Han E, Klein KC. Pre-class learning methods for flipped classrooms[J].Am J Pharm Educ, 2019,83(1):6922.

14. Persky AM, Hogg A. Influence of reading material characteristics on study time for pre-class quizzes in a flipped classroom[J].Am J Pharm Educ, 2017,81(6):103.

15. Francis N, Morgan A, Holm S, Davey R, Bodger O, Dudley E. Adopting a flipped classroom approach for teaching molar calculations to biochemistry and genetics students[J].Biochem Mol Biol Educ, 2019,48(3):220-6.

16. Medina MS, Conway SE, Davis-Maxwell TS, Webb R. The impact of problem solving feedback on team-based learning case responses[J].Am J Pharm Educ, 2013,77(9):189.

17. Davies RS, Dean DL, Ball N. Flipping the classroom and instructional technology integration in a college-level information systems spreadsheet course[J].Educ Technol Res Dev, 2013,61(4):563-80.

18. RTN W-YIPRR, Chung TK, Tang CS. The Chinese Childbirth Self-Efficacy Inventory: the development of a short form[J]. Journal of Clinical Nursing, 2008, 17(3): 333-340.

19. Xiaona DAI, CHEN Xu, XU Ling. The application of breastfeeding confidence scale in nursing practice [J]. Chinese Journal of Nursing, 2004, 39(6): 407-409.

20. Zung WW. A rating instrument for anxiety disorders[J]. Psychosom, 1971,12(6): 371-379.

21. ZUNG WW. A self-rating depression scale[J]. Arch Gen Psy-chiatry,1965,12: 63-70. 
22. Gagnon AJ, Sandall J. Individual or group antenatal education for childbirth or parenthood, or both[J]. Cochrane Database Syst Rev, 2007(3):CD002869.

23. Ahldén I, Ahlehagen S, Dahlgren LO, Josefsson A.Parents' expectations about participating in antenatal parenthood education classes[J]. J Perinat Educ, 2012,21(1):11-17.

24. Canoso JJ, Saavedra MÁ, Pascual-Ramos V,Sánchez-Valencia MA,Kalish RA. Musculoskeletal anatomy by self-examination: a learner-centered method for students and practitioners of musculoskeletal medicine[J]. Ann Anat, 2020,228:151457.

25. Matsuyama Y, Nakaya M, Okazaki H, Lebowitz AJ, Leppink J, van der Vleuten C. Does changing from a teacher-centered to a learner-centered context promote self-regulated learning: a qualitative study in a Japanese undergraduate setting[J]. BMC Med Educ, 2019,19(1):152.

26. Petersson, K., Petersson, C. \& Hakansson, A. (2004) What is good parental education?[J]. Nordic College of Caring Sciences, 18, 82-89.

27. P. Pålsson, L.J. Kvist, M. Ekelin, I.K. Hallström, E.K. Persson, “I didn’t know what to ask about": first-time mothers' conceptions of prenatal preparation for the early parenthood period[J]. Perinatal Educ, 27 (3) (2018) 163.

28. ZHU Xin-li, GU Chun-yi, TAO Hua, et al. Survey on normal primiparae's expected mode of delivery and its influencing factors under two-child policy[J]. Chin J Nurs, September 2016,51:1044-48.

29. Maimburg RD, Vaeth M, Durr J, et al. Randomised trial of structured antenatal training sessions to improve the birth process[J]. BJOG, 2010,117:921-8.

30. Taheri Z, Mazaheri MA, Khorsandi M, et al. Effect of educational intervention on self-efficacy for choosing delivery method among pregnant women in 2013[J]. Int J Prev Med, 2014,5:1247-54.

31. Ip WY, Tang CS, Goggins WB. An educational intervention to improve women's ability to cope with childbirth[J]. J Clin Nurs, 2009,18:2125-35

32. Institute of Medicine (US) and National Research Council. (US) Committee to Reexamine IOM Pregnancy Weight Guidelines; Rasmussen KM, Yaktine AL, editors. Weight Gain During Pregnancy: Reexamining the Guidelines[J]. Washington (DC): National Academies Press (US),2009.

33. Tzoulaki I, Sovio U, Pillas D, Hartikainen AL, Pouta A, LaitinenJ, Tammelin TH, Jarvelin MR, Elliott, P. Relation of immediate postnatal growth with obesity and related metabolic risk factors in adulthood: the northern Finland birth cohort 1966 study[J]. Am J Epidemiol, 2010,171(9):989-98.

34. Yong, Heng Yaw, Mohd Shariff, Zalilah, et al. Higher Parity, Pre-Pregnancy BMI and Rate of Gestational Weight Gain Are Associated with Gestational Diabetes Mellitus in Food Insecure Women[J]. Int J Environ Res Public Health, 2021,18,2694. 


\section{Figure Legends}

Figure 1. Blended learning design including the FC with TBL approach used in this study.

Table 1. Baseline characteristics of participants between the two groups.

Table 2. Differences in learning outcome and psychological tests in both groups. 
Table 3. Comparison of GWG and childbirth between the two groups.

Table 4. Comparison of GWG between the two groups in different periods.

Table 5. Comparison of labor duration between two groups of vaginal delivery.

Table 6. Comparison of the test scores of CSE and BSE between the two groups.

Table 7. Comparison of complications during childbirth between the two groups. 


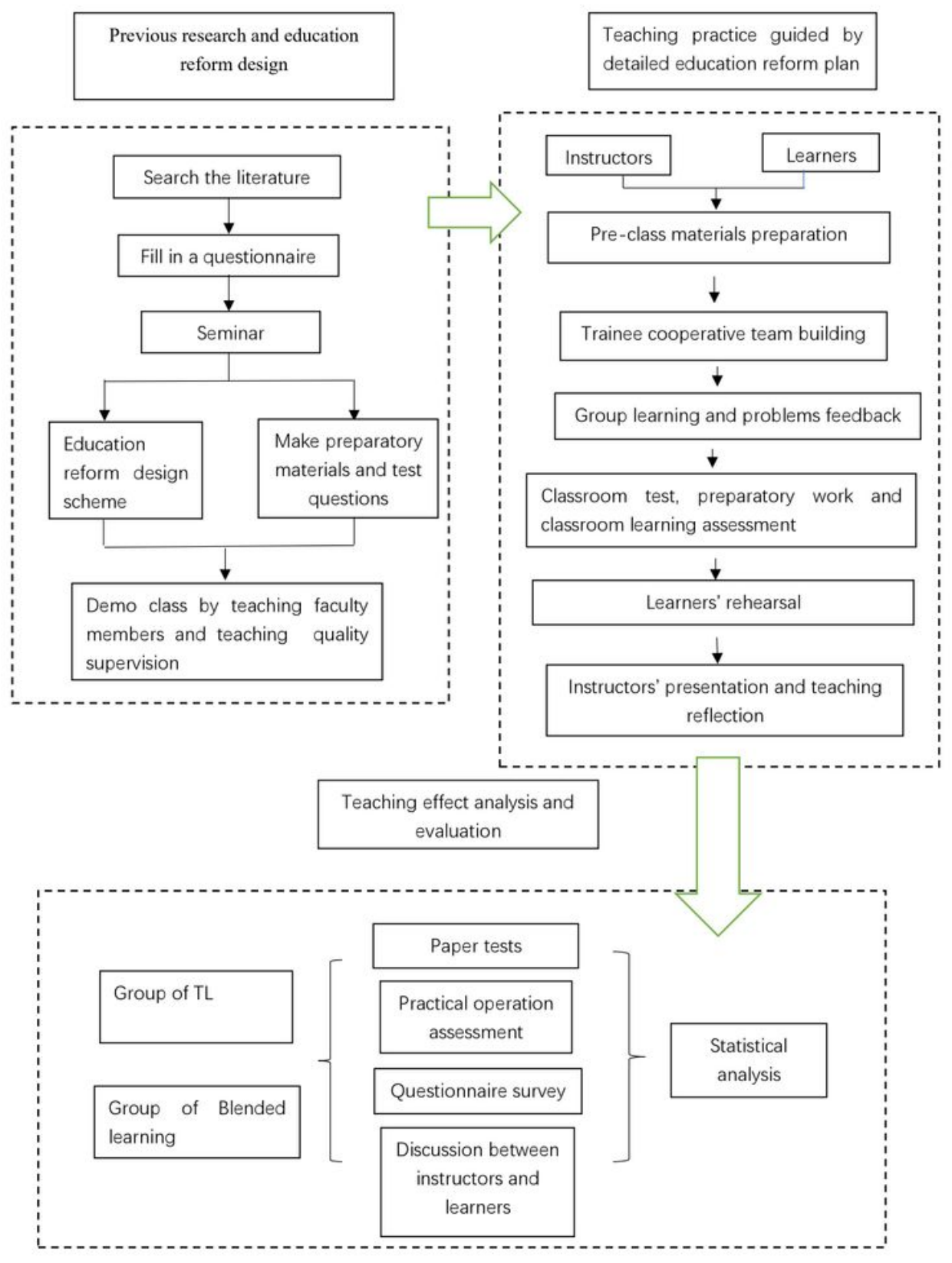

Figure 1

Blended learning design including the FC with TBL approach used in this study. 